\title{
Exploring the Neural Basis of Cognitive Reserve*
}

\author{
Yaakov Stern, Eric Zarahn, H. John Hilton, Joseph Flynn, Robert DeLaPaz, and Brian Rakitin \\ Cognitive Neuroscience Division, Departments of Neurology, Psychiatry and Radiology, Taub Institute \\ for Research in Alzheimer's Disease and the Aging Brain and College of Physicians and Surgeons, \\ Columbia University, New York, NY, USA
}

\begin{abstract}
There is epidemiologic and imaging evidence for the presence of cognitive reserve, but the neurophysiologic substrate of CR has not been established. In order to test the hypothesis that CR is related to aspects of neural processing, we used fMRI to image 19 healthy young adults while they performed a nonverbal recognition test. There were two task conditions. A low demand condition required encoding and recognition of single items and a titrated demand condition required the subject to encode and then recognize a larger list of items, with the study list size for each subject adjusted prior to scanning such that recognition accuracy was $75 \%$. We hypothesized that individual differences in cognitive reserve are related to changes in neural activity as subjects moved from the low to the titrated demand task. To test this, we examined the correlation between subjects' fMRI activation and NART scores. This analysis was implemented voxel-wise in a whole brain fMRI dataset. During both the study and test phases of the recognition memory task we noted areas where, across subjects, there were significant positive and negative correlations between change in activation from low to titrated demand and the NART score. These correlations support our hypothesis that neural processing differs across individuals as a function of CR. This differential processing may help explain individual differences in capacity, and may underlie reserve against age-related or other pathologic changes.
\end{abstract}

The theory of cognitive reserve $(\mathrm{CR})$ posits that there is not a direct relationship between the severity of neural effect of any pathologic insult and its effect on cognitive function. Rather the effect of the brain pathology is mediated through reserve, such that in individual with more reserve will be able to withstand more pathology before performance is affected (Stern, 2002). Thus, several epidemiologic studies have suggested differential susceptibility to age-related memory changes and Alzheimer's disease (AD) is related to variables associated with $\mathrm{CR}$, such as education, occupation (Evans et al., 1993; Letenneur, Commenges, Dartigues, \& Barberger-Gateau, 1994; Stern et al., 1994; White et al., 1994) or leisure activities (Fabrigoule et al., 1995; Friedland, Fritsch, Smyth, et al., 2001; Kondo,
Niino, \& Shido, 1994; Scarmeas, Levy, Tang, Manly, \& Stern, 2001; Wilson et al., 2002). These observations are complemented by imaging studies of resting brain metabolism, which suggest that AD patients with higher CR can operate at the same level of clinical severity as patients with lower CR while suffering from more advanced AD pathology (Alexander et al., 1997; Scarmeas et al., in press; Stern, Alexander, Prohovnik, \& Mayeux, 1992; Stern et al., 1995). In two of these studies, proxies for CR included measures of word reading (which themselves are considered to be good estimates of Verbal IQ). While these studies and others provide support for the concept of CR, the neurophysiologic substrate of $\mathrm{CR}$ has not been established. We have suggested an active form of $\mathrm{CR}$, where differential $\mathrm{CR}$ is associated with

\footnotetext{
* Supported by NIA Grant AG 14671.

Address correspondence to: Yaakov Stern, 630 West 168th Street, P\&S Box 16, New York, NY 10032, USA. Tel.: +1-212-305-9194. Fax: +1-212-305-2426. E-mail: ys11@ columbia.edu

Accepted for publication: December 25, 2002.
} 
different ways of processing task demands (Stern, 2002). For example, higher levels of CR may be associated with more efficient synaptic processing. This predicts that task-related neural processing in individuals of a given age would be a function of CR. These neurophysiologic differences across subjects should be present not only in individuals affected by brain pathology, but even in healthy, young individuals. Further, testing for such relationships in young subjects has an advantage in that one need not be concerned with the confound of across-subject variability in agerelated brain pathology.

In order to test the hypothesis that $\mathrm{CR}$ is related to aspects of neural processing, we imaged healthy young adults while they performed a nonverbal recognition test. There were two task conditions. A low demand condition required encoding and recognition of single items. A titrated demand condition required the subject to encode and then recognize a larger list of items. Prior to scanning, the study list size (SLS) of the titrated demand condition was adjusted for each subject such that recognition accuracy was $75 \%$. This procedure was intended to match task difficulty (as operationalized by performance) across subjects.

Our intention was to explore how individual differences in cognitive reserve are related to changes in neural activity as subjects moved from the low to the titrated demand task. A prediction of the hypothesis stated above was that certain aspects of this activation, as measured by fMRI, would be related to measures of cognitive reserve. We simplified this prediction to that of a correlation between fMRI activation and CR measures (IQ measures). This analysis was done voxel-wise in a whole brain fMRI dataset. If there were areas of the brain that expressed such a correlation, they would be candidates for regions/ systems that were especially related to the neural instantiation of $\mathrm{CR}$.

\section{METHODS}

Nineteen healthy young adults, between the ages of 18 and 30, participated. All were carefully screened to insure that they had no neurological or psychiatric disease. All subjects were right-handed. Informed consent was obtained after the nature and risks of the study were explained.

We used the raw score of the New Adult Reading Test (NART) (Nelson, 1982) and WAIS-R Vocabulary (Wechsler, 1981) age scaled scores as proxy measures for cognitive reserve. These tests are considered to be a good estimates of Verbal IQ (Grober \& Sliwinski, 1991; Nelson \& O'Connell, 1978). We also considered using years of education as an additional proxy measure, but the range of educational attainment was severely limited in this group of young subjects (16 of 19 had completed 16 years of education).

\section{Nonverbal Recognition Task}

Two conditions of a nonverbal recognition test were considered in the current analysis. The basic task consisted of the serial presentation of one or more single uncommon shapes, followed by a series of the same number of recognition test probes (Fig. 1). The test items were distinguished from study items by
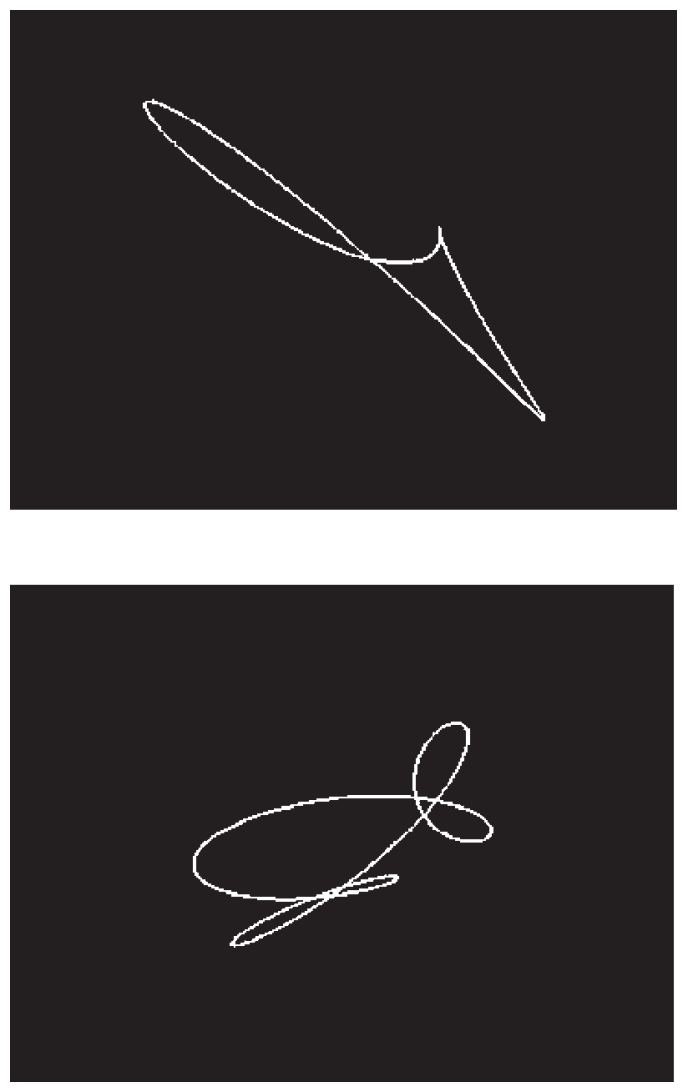

Fig. 1. Sample of shapes used for the recognition task. 
a circumscribed rectangular frame. Subjects were instructed to make a "new" or "old" response for each test item by pressing with the right or left finger, respectively, on a LUMI touch response device. Instructions were to stress accuracy over speed. New and old test probes occurred with the frequency of $50 \%$. Test items were psuedo-randomized so that no more than four consecutive trials required the same response.

Each shape was used only once for each subject. A set of shapes was designed to have similar characteristics that varied randomly within given sets of parameters. Loop shapes were used since their level of complexity made verbal encoding difficult (Fig. 1). The stimuli were pre-screened to insure that they could not easily be given a name.

In the low demand condition, the SLS was 1 (i.e., one study shape followed by one recognition probe). The titrated demand condition used a subject-specific SLS that was predetermined to elicit a recognition accuracy of $75 \%$. Prior to the fMRI scan, each subject completed a standardized training session and then two 15-min titration sessions. During these sessions, the particular SLS value at which the subject achieved 75\% accuracy was determined using a staircase method of SLS adjustment. This SLS value was then used in the titrated demand condition on the day of the scan.

fMRI data for all conditions were acquired over three separate sessions (i.e., runs). In each session, the titrated demand condition lasted $240 \mathrm{~s}$, and the lowdemand condition (seven trials) $112 \mathrm{~s}$.

Each low-demand trial was 16-s long: Each trial began with a 600-ms ITI, followed by the studied stimulus for $3400 \mathrm{~ms}$, the test item for an additional 3400 , followed by an $8600 \mathrm{~ms}$ resting baseline. ${ }^{1}$

For the titrated condition, the number of study and test presentations was the SLS established for each subject. Study and test periods repeated as many times as possible (depending on the SLS) over the course of the 240-s interval, with new study and test lists presented on each iteration. Each titrated demand trial began with a 600-ms ITI followed sequentially by stimuli for $3400 \mathrm{~ms}$ stimulus presentations. Subjects were instructed to study the object for the entire $3400 \mathrm{~ms}$ on study trials. Presentation of the test list (3400 ms per test stimulus) followed immediately after presentation of the study list.

\footnotetext{
${ }^{1}$ The purpose of the baseline was to increase signal: noise for the comparison of titrated and low demand. Though this might seem nonintuitive, it is related to the low frequency noise seen in fMRI data. Baseline periods were not similarly intercalated into the titrated demand condition because that would have disturbed its psychological structure.
}

\section{fMRI Acquisition and Processing}

Functional images were acquired using a $1.5 \mathrm{~T}$ magnetic resonance scanner (General Electric) retrofitted for echoplaner imaging. A gradient echo EPI sequence $\left(\mathrm{TE}=50 \mathrm{~ms} ; \mathrm{TR}=3 \mathrm{~s}\right.$; flip angle $\left.=90^{\circ}\right)$ and a standard quadrature head coil was used to acquire T2* weighted images with an in-plane resolution of $3.124 \mathrm{~mm} \times 3.124 \mathrm{~mm}\left(64 \times 64\right.$ matrix; $20 \mathrm{~cm}^{2}$ field of view). Based on $\mathrm{T}_{1}$ "scout" images, $8 \mathrm{~mm}$ transaxial slices (15-17) were acquired. Following the fMRI runs, a high (in-plane) resolution $\mathrm{T}_{2}$ image at the same slice locations used in the fMRI run was acquired using a fast spin echo sequence $(\mathrm{TE}=105 \mathrm{~ms} ; \mathrm{TR}=2500 \mathrm{~ms}$; $256 \times 256$ matrix; $20 \mathrm{~cm}^{2}$ field of view).

All image processing and analysis was done using the SPM99 program (Wellcome Department of Cognitive Neurology) and other code written in Matlab 5.3 (Mathworks, Natic, MA). fMRI time series were corrected for order of slice acquisition. All functional volumes in a given subject were realigned to the first volume from the first run of each study. The $T_{2}$ anatomical image was then co-registered to the first functional volume, using the mutual information coregistration algorithm implemented in SPM99. This co-registered structural image was then used in determining nonlinear spatial normalization $(7 \times 8 \times 7$ nonlinear basis functions) parameters for a transformation into a Talairach standard space defined by the Montreal Neurological Institute template brain applied with SPM 99. These normalization parameters were then applied to the functional data (using sinc-interpolation to reslice the images to $2 \mathrm{~mm} \times 2 \mathrm{~mm} \times 2 \mathrm{~mm}$ ).

\section{Data Analysis}

The schemes of the fMRI analyses comprised two levels (Holmes \& Friston, 1998). The first involved time series modeling (Worsley \& Friston, 1995) and generating contrast images of interest for each study subject. The second involved hypothesis testing at the population-level (Friston, Holmes, Price, Buchel, \& Worsley, 1999).

At the time-series modeling stage, the fMRI responses to stimulus presentation in each condition (i.e., either low or titrated-demand) and period (i.e., either study or test) were modeled with appropriately timed rectangular $3400 \mathrm{~ms}$ pulses convolved with a canonical hemodynamic response function (the default in SPM99). In the first level of analysis, contrast images, which compare the amplitudes of these fMRI responses, were estimated in each subject. An example of a specific contrast is the amplitude of the titrated demand, test period response minus that of the low demand, test period response. Prior to population hypothesis testing these contrast images were divided by the mean EPI image for that subject (i.e., they were intensity-normalized) in order to eliminate artifactual 
differences in signal range across datasets due to scanner calibration/data reconstruction issues. The intensity normalized images were then spatially smoothed with an isotropic Gaussian kernel (fullwidth-at-half-maximum $=8 \mathrm{~mm}$ ).

The second level of voxel-wise analysis assessed the relationship of our measures of $\mathrm{CR}$ to differences between the low and titrated demand event-related fMRI response amplitudes. These analyses were performed separately for the study and test periods. These regression analyses compute error terms on unexplained inter-subject variation and effects, thus providing for inference at the population level (Holmes \& Friston, 1998). The mapwise false-positive rate for the resulting $\operatorname{SPM}\{t\}$ maps was controlled at approximately $\alpha=0.05$ per hypothesis using Bonferroni correction for the number of resolution elements.

Even though the purpose of the titration procedure was to constrain accuracy at $75 \%$ in the titrated demand condition, there were residual variations from this target value. Therefore, recognition accuracy (percent correct) for the titrated demand condition was included as a covariate to obviate confounding of the reserve variables and task performance. Titrated demand SLS (orthogonalized with respect to the other variables) was also initially included as a covariate to explain other possible variance components of the data in order to increase sensitivity.

Model reduction was attempted by assessing the relative effects sizes associated with the different covariates. In the context of this full regression model, those covariates which produced negative results with the voxel-wise tests were subjected to a global test (Worsley et al., 1997). This test has no spatial localizing power, but is more sensitive than voxel-wise tests to spatially diffuse, low intensity effects. A negative result with the global test provides more confidence than any lack of a detectable effect at the voxel-wise level is not due to high Type 2 error for spatially diffuse effects. When these global tests were also negative, the covariate was eliminated from the regression model. This approach eliminated recognition accuracy, SLS, and WAIS-R Vocabulary. Further inspection of the results suggested that a quadratic term for NART decreased residual variability. Thus, the final model consisted solely of an overall intercept, and orthogonal linear and quadratic terms for NART.

\section{RESULTS}

\section{Subject Demographics and Behavioral Performance}

Mean education was 15.6 (Table 1); all but three subjects had 16 years of education. Performance
Table 1. Subject Demographics, and Performance on the Two Shape Recognition Task Conditions.

\begin{tabular}{lrr}
\hline & \multicolumn{1}{c}{ Mean } & \multicolumn{1}{c}{$S D$} \\
\hline Education & 15.58 & 1.43 \\
WAIS-R Vocabulary & 13.16 & 1.77 \\
NART IQ & 120.66 & 5.36 \\
Low demand & & \\
$\%$ correct & 91.98 & 5.03 \\
RT (correct responses) & 1059.48 & 371.06 \\
Titrated demand & & \\
Study list size & 20.16 & 8.45 \\
$\%$ correct & 75.84 & 10.27 \\
RT (correct responses) & 1544.91 & 374.53 \\
\hline
\end{tabular}

accuracy was quite good on the low demand task. As intended, the titration procedures prior to the scan session produced a performance accuracy of approximately $75 \%$ on the titrated demand condition during the fMRI session.

\section{fMRI Data}

\section{Study}

During study, activation was greater in titrated minus demand than in low demand in the following areas: left and right caudate (tail) and insula; left superior frontal gyrus, inferior parietal lobule, and medial frontal gyrus; and right precentral gyrus, superior frontal gyrus, cingulate gyrus, and superior temporal gyrus (Table 2 and Fig. 2).

Positive correlations between titrated-low demand and NART were seen in left middle frontal gyrus and negative correlations were seen in right superior frontal gyrus, middle frontal gyrus, precentral gyrus, medial frontal gyrus, and insula (Table 2 and Fig. 3).

Test

During test, activation was greater in titrated demand than in low demand in the following areas: left and right precentral gyrus; left middle frontal gyrus, fusiform gyrus, cuneus, inferior occipital gyrus, caudate (tail); and right inferior temporal and fusiform gyrus (Table 3 and Fig. 4).

Positive correlations between titrated-low demand and NART were seen in left postcentral gyrus, and right medial frontal gyrus. Negative correlations were seen in many areas, including 
Table 2. Maxima of Areas of Significant Activation During the Study Phase of the Task.

\begin{tabular}{|c|c|c|c|c|c|c|}
\hline \multirow[t]{2}{*}{ Contrast } & \multicolumn{4}{|c|}{ Talairach coordinates } & \multirow[t]{2}{*}{ Location } & \multirow[t]{2}{*}{ BA } \\
\hline & $x$ & $y$ & $z$ & $T$ value & & \\
\hline \multirow{13}{*}{$\begin{array}{l}\text { Mean effect: titrated } \\
\text { minus low demand }\end{array}$} & -22 & -40 & 11 & 6.67 & Left caudate tail & \\
\hline & 57 & -4 & 28 & 6.63 & Right precentral gyrus & 6 \\
\hline & 6 & 58 & 36 & 5.85 & Right superior frontal gyrus & 9 \\
\hline & 28 & -28 & 18 & 5.8 & Right insula & 13 \\
\hline & 20 & -38 & 24 & 5.61 & Right cingulate gyrus & 31 \\
\hline & -42 & -15 & 8 & 5.29 & Left insula & 13 \\
\hline & -12 & 65 & 17 & 5.21 & Left superior frontal gyrus & 10 \\
\hline & 38 & -48 & 6 & 5.17 & Right superior temporal gyrus & 39 \\
\hline & 38 & -21 & 1 & 4.99 & Right insula & 13 \\
\hline & -55 & -58 & 38 & 4.85 & Left inferior parietal lobule & 40 \\
\hline & -28 & -50 & 14 & 4.76 & Left temporal lobe (subgyral) & \\
\hline & 22 & -15 & 43 & 4.7 & Right cingulate gyrus & 24 \\
\hline & -16 & 58 & 3 & 4.68 & Left medial frontal gyrus & 10 \\
\hline Positive correlation with NART & -20 & 54 & -11 & 7.06 & Left middle frontal gyrus & 10 \\
\hline \multirow[t]{10}{*}{ Negative correlation with NART } & 8 & 58 & 36 & 8.34 & Right superior frontal gyrus & 9 \\
\hline & 20 & 56 & 3 & 6.37 & Right superior frontal gyrus & \\
\hline & 16 & 66 & 2 & 5.47 & Right superior frontal gyrus & 10 \\
\hline & 30 & 47 & 1 & 5.8 & Right middle frontal gyrus & \\
\hline & -26 & 43 & 3 & 5.78 & Left subgyral & \\
\hline & 55 & -10 & 30 & 5.69 & Right precentral gyrus & 4 \\
\hline & 40 & -25 & 1 & 5.57 & Right insula & \\
\hline & 24 & 47 & 12 & 5.09 & Right medial frontal gyrus & 10 \\
\hline & -6 & -39 & -37 & 4.99 & Left medulla & \\
\hline & 8 & -50 & -34 & 4.71 & Right cerebellum & \\
\hline
\end{tabular}

Note. The mean effect summarizes areas where activation was higher in the titrated demand than in the low demand task. There were no areas where activation in low demand was higher. The correlations summarize areas where differences in titrated - low demand across subjects correlated with NART scores. Both positive and negative correlations were noted.

left and right medial frontal gyrus, superior frontal gyrus, postcentral gyrus, and precentral gyrus; left middle temporal gyrus, superior temporal gyrus, thalamus, superior parietal lobule, and parahippocampal gyrus; and right inferior parietal lobule, insula, precentral gyrus, and claustrum (Table 3 and Fig. 4).

\section{DISCUSSION}

We tested the idea that $\mathrm{CR}$ is associated with neurophysiologic differences in task processing. To do this we explored how individual differences in $\mathrm{CR}$ are related to changes in neural activity as subjects moved from the low to the titrated demand task. We predicted that certain aspects of this activation, as measured by fMRI, would be related to measures of CR. The primary finding of this study was that, both during study and during subsequent retrieval, brain areas were noted where there was a systematic relationship between CR and brain activation. These correlations support our hypothesis that neural processing differs as a function of CR. This differential processing may help explain individual differences in capacity, and may underlie reserve against age-related or other pathologic changes.

We felt that it was important to control for task difficulty in the titrated demand task, as measured by recognition accuracy. Several studies have 

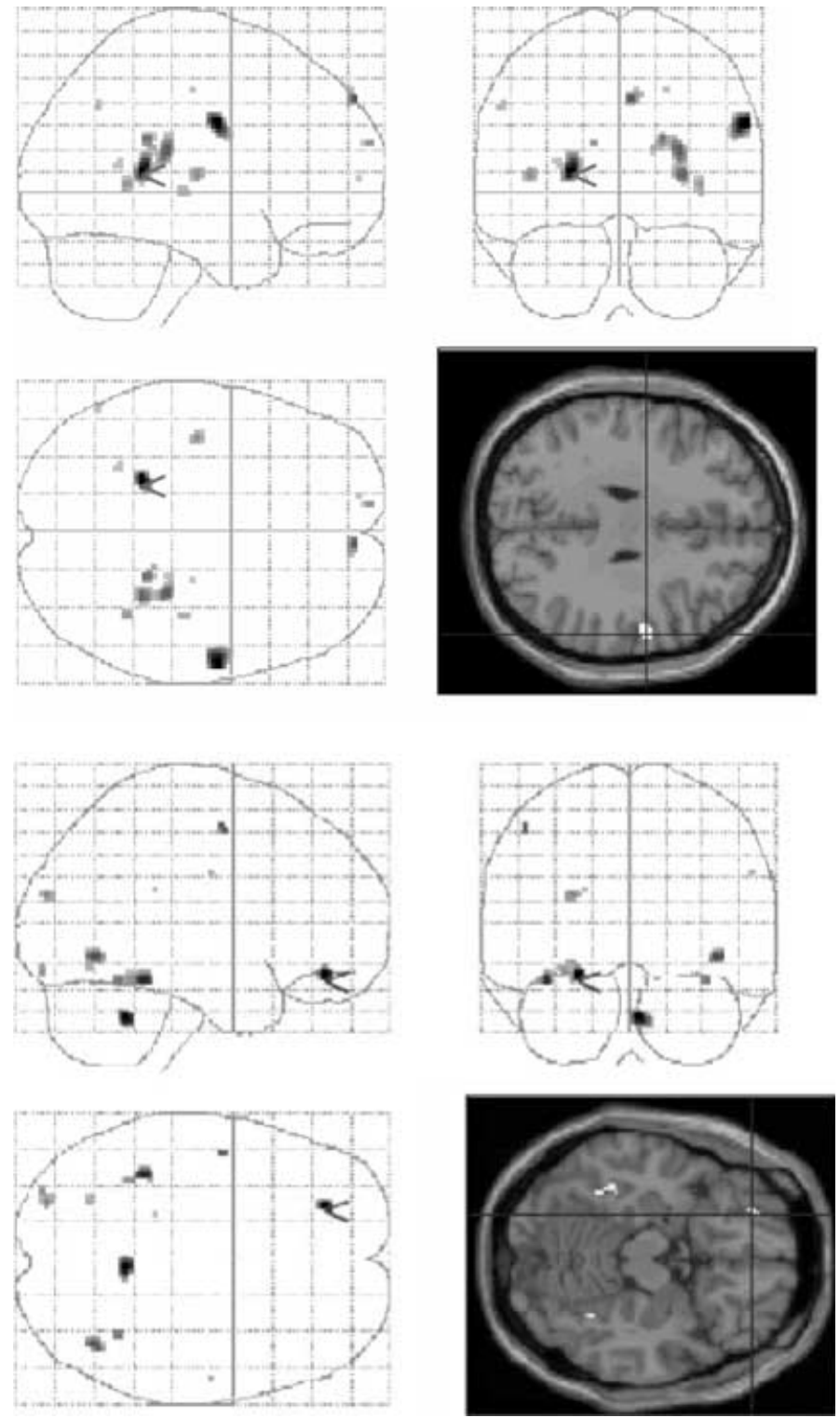

Fig. 2. Mean difference in activation in the high versus low demand conditions. Top is during study, bottom is during test.

suggested that differential recruitment may be a function of differences in task difficulty (Price \& Friston, 2001; Reuter-Lorenz et al., 2000, 2001; Stern, 2002; Stern et al., 2000). This might suggest that subjects with more reserve show less activation. However the direction of these differences could be a function of the activation task methodology. Since any task would be more difficult for individuals with lower IQ, it is more likely to elicit more extensive activation in those individuals. The possibility would then remain that patterns of activation would not differ if difficulty were held constant. By matching task difficulty across subjects, we can be more confident that differences in brain activation across subjects is not simply a function differences in 

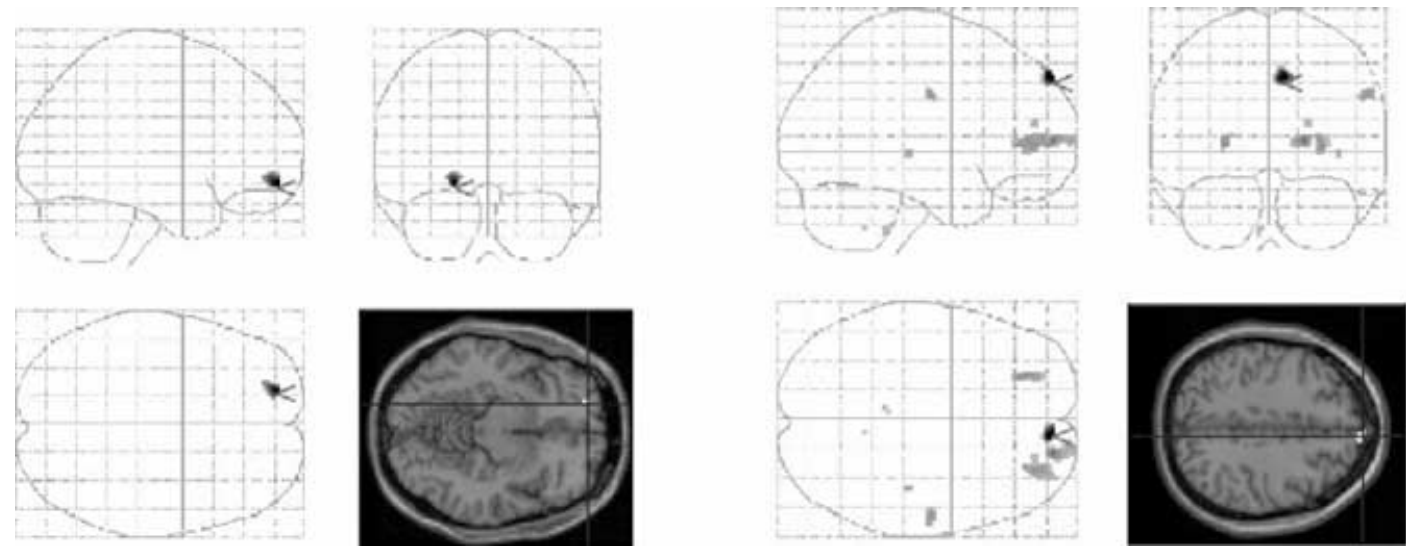

Titrate Study - Low Study NART correct at [-20 56 -10]

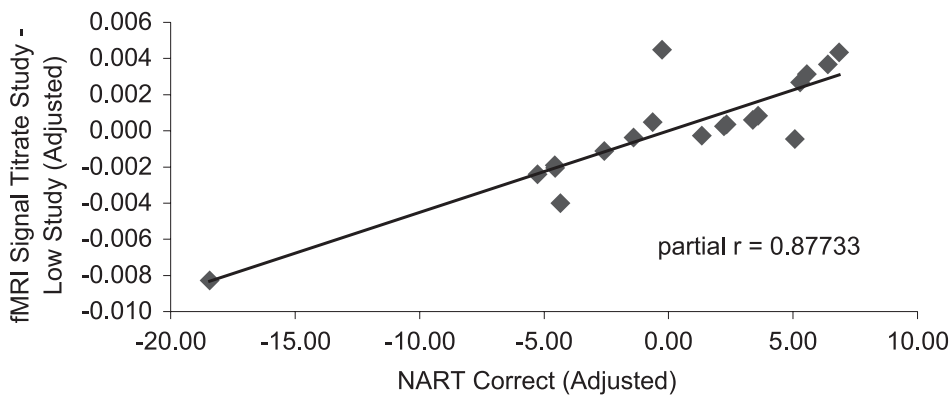

Titrate Study - Low Study NART correct at [8 58 42]

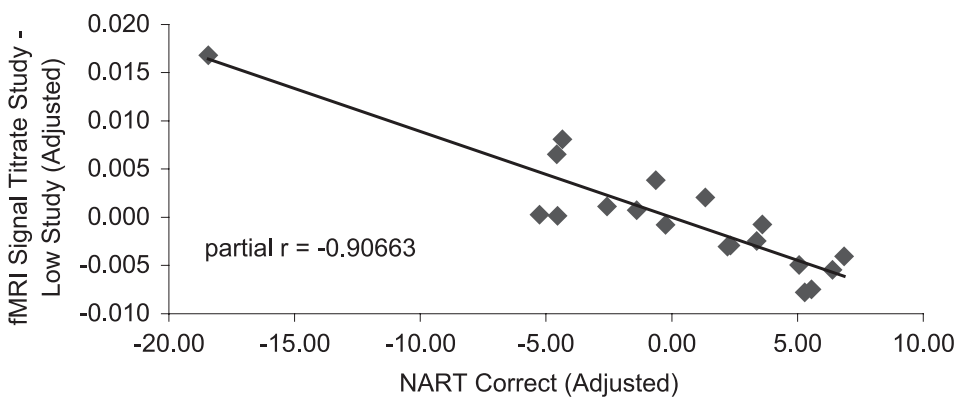

Fig. 3. Areas where titrate minus low demand activation during study correlated positively (left) and negatively (right) with NART score. Scatter graphs below illustrate these correlations at a selected voxel.

how difficult that task was for each subject. Rather, the differential activation is more likely to reflect actual differences in neural network underlying task performance.

Even though subjects were given memory loads that were tailored to their performance during a titration session, there was still variability in recognition performance during fMRI scanning. However, like SLS, recognition performance during fMRI scanning was weakly correlated with NART $(r=.09 ;$ n.s. $)$, and had no detectable effects in the neuroimaging data. Thus, it does not seem possible that the relationship observed between fMRI responses and NART are an artifact of correlations with recognition performance. 
Table 3. Maxima of Areas of Significant Activation During the Test Phase of the Task.

\begin{tabular}{|c|c|c|c|c|c|c|}
\hline \multirow[t]{2}{*}{ Contrast } & \multicolumn{4}{|c|}{ Talairach coordinates } & \multirow[t]{2}{*}{ Location } & \multirow[t]{2}{*}{ BA } \\
\hline & $x$ & $y$ & $z$ & $T$ value & & \\
\hline \multirow{11}{*}{$\begin{array}{l}\text { Mean effect: titrated minus } \\
\text { low demand }\end{array}$} & -24 & 40 & -15 & 5.79 & Left middle frontal gyrus & 11 \\
\hline & 4 & -50 & -26 & 5.72 & Right cerebellum & \\
\hline & -38 & -41 & -13 & 5.41 & Left fusiform gyrus & 20 \\
\hline & 40 & -62 & -2 & 5.18 & Right inferior temporal gyrus & \\
\hline & -48 & -1 & 48 & 5.12 & Left precentral gyrus & 6 \\
\hline & 36 & -51 & -13 & 4.95 & Right fusiform gyrus & 37 \\
\hline & -26 & -82 & 24 & 4.85 & Left cuneus & 19 \\
\hline & -30 & -86 & -7 & 4.8 & Left inferior occipital gyrus & 18 \\
\hline & -26 & -64 & -5 & 4.62 & Left fusiform gyrus & 19 \\
\hline & 55 & -8 & 30 & 4.59 & Right precentral gyrus & 6 \\
\hline & -20 & -34 & 24 & 4.56 & Left caudate tail & \\
\hline \multirow[t]{2}{*}{ Positive correlation with NART } & -48 & -15 & 54 & 5.79 & Left postcentral gyrus & $J$ \\
\hline & 16 & 48 & -6 & 5.29 & Right medial frontal gyrus & 10 \\
\hline \multirow[t]{23}{*}{ Negative correlation with NART } & -55 & -35 & 4 & 6.68 & Left middle temporal gyrus & 22 \\
\hline & -46 & -33 & 9 & 5.07 & Left superior temporal gyrus & 41 \\
\hline & 16 & 68 & 2 & 6.14 & Right superior frontal gyrus & 10 \\
\hline & 24 & 49 & 10 & 6 & Right medial frontal gyrus & 10 \\
\hline & -16 & 49 & 9 & 5.84 & Left medial frontal gyrus & 10 \\
\hline & 57 & 4 & 33 & 5.58 & Right precentral gyrus & 6 \\
\hline & -30 & -60 & 14 & 5.5 & Left middle temporal gyrus & 19 \\
\hline & -24 & 42 & -16 & 5.5 & Left superior frontal gyrus & 11 \\
\hline & 63 & -34 & 24 & 5.43 & Right inferior parietal lobule & 40 \\
\hline & -36 & -34 & 64 & 5.43 & Left postcentral gyrus & 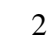 \\
\hline & -26 & -12 & 67 & 5.32 & Left precentral gyrus & 6 \\
\hline & -18 & -35 & 9 & 5.32 & Left thalamus & \\
\hline & -22 & -55 & 65 & 5.22 & Left superior parietal lobule & $r$ \\
\hline & 55 & -36 & 15 & 5.13 & Right insula & 13 \\
\hline & 42 & -42 & 61 & 5.08 & Right postcentral gyrus & 5 \\
\hline & -63 & -27 & 40 & 5.05 & Left postcentral gyrus & 2 \\
\hline & -48 & -44 & 19 & 4.91 & Left superior temporal gyrus & 13 \\
\hline & 30 & -10 & 65 & 4.89 & Right precentral gyrus & 6 \\
\hline & -14 & -14 & -11 & 4.75 & Left parahippocampal gyrus & 37 \\
\hline & 32 & -12 & 63 & 4.62 & Right precentral gyrus & 6 \\
\hline & 38 & -25 & -2 & 4.59 & Right claustrum & \\
\hline & 55 & -19 & 38 & 4.56 & Right postcentral gyrus & 3 \\
\hline & 22 & -26 & 71 & 4.56 & Right precentral gyrus & 4 \\
\hline
\end{tabular}

Note. The mean effect summarizes areas where activation was higher in the titrated demand than in the low demand task. There were no areas where activation in low demand was higher. The correlations summarize areas where differences in titrated - low demand across subjects correlated with NART scores. Both positive and negative correlations were noted.

It could be argued that an inherent weakness of an experimental design that controls for task difficulty is that it inevitably results in different subjects engaging different levels or intensities of cognitive processing. From a theoretical point of view, this argument, though intuitively compel- ling, is self-contradicting in its most primitive interpretation. If difficulty (as measured by \% correct accuracy) is the critical variable to equate between subjects, then this implies that the value of superficial task parameters (e.g., the size of the shape list) per se is not. On an empirical level, 

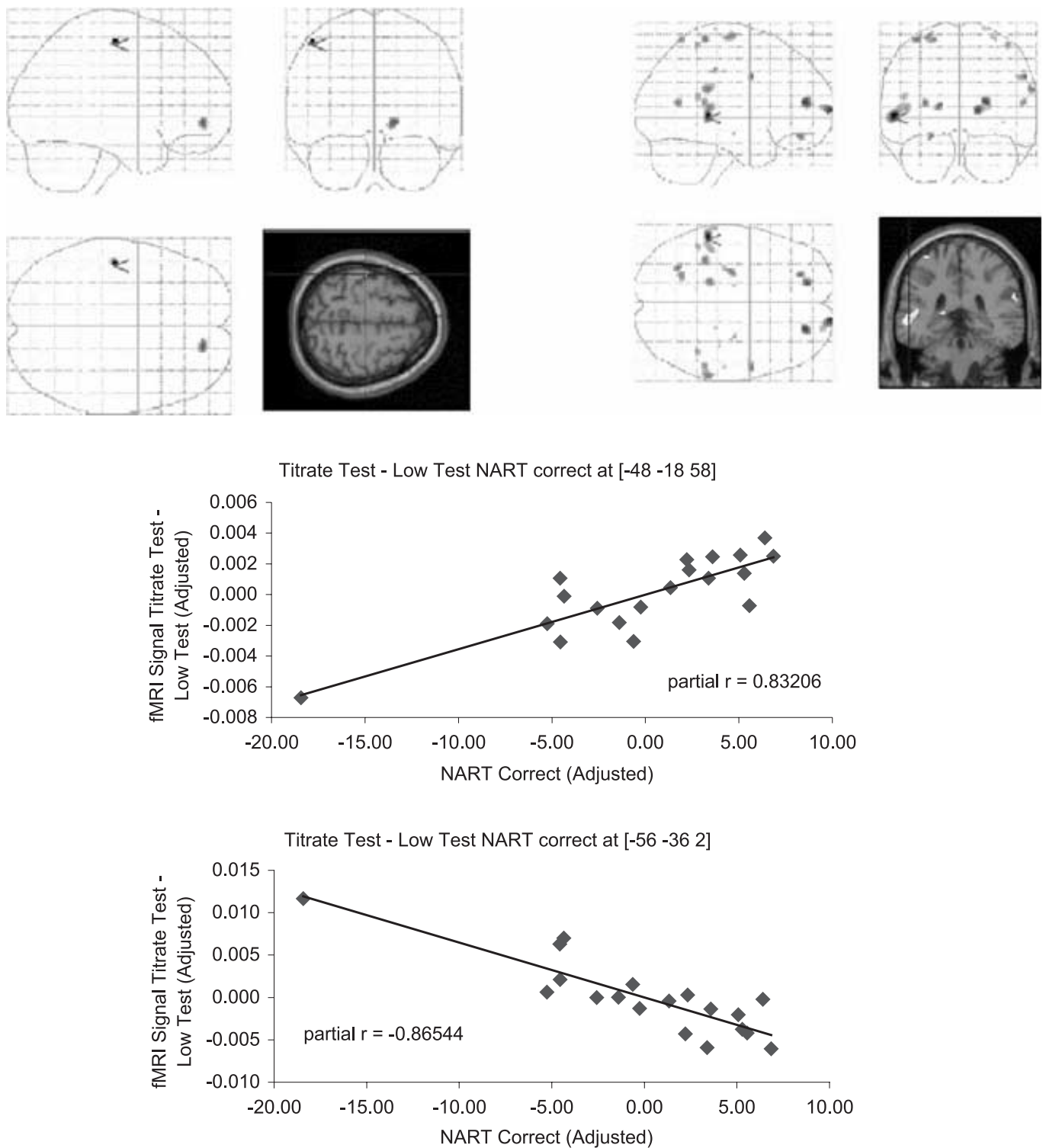

Fig. 4. Areas where titrate minus low demand activation during test correlated positively (left) and negatively (right) with NART score. Scatter graphs below illustrate these correlations at a selected voxel.

although there was a large degree of variability in the SLS, SLS had no detectable effects in the neuroimaging data. Also, the correlation of SLS and NART was weak $(r=.22$, n.s.). Therefore, it is not tenable that the correlations with NART are actually due to the variability in SLS across subjects.

The weak correlation between SLS and NART also suggests that an individual's level of CR is not synonymous with their memory ability. Further, in our analyses SLS was not related to the change in activation from low to titrated demand. As implemented, the memory task provided a cognitive challenge whose difficulty was comparable across subjects. This response to increased task demand was more related to an index of CR that to the titrated SLS. This is consonant with a view of CR as allowing an individual to cope with 
challenges in general, as opposed to CR simply being synonymous with better performance in a single cognitive domain (such as memory).

In summary, these data suggest that individual differences in CR are related to changes in neural activity as subjects moved from the low to the titrated demand task. The areas of the brain that expressed a correlation between fMRI activation and the NART are candidates for regions/systems that are especially related to the neural instantiation of CR.

\section{REFERENCES}

Alexander, G.E., Furey, M.L., Grady, C.L., Pietrini, P., Mentis, M.J., \& Schapiro, M.B. (1997). Association of premorbid function with cerebral metabolism in Alzheimer's disease: Implications for the reserve hypothesis. American Journal of Psychiatry, 154, 165-172.

Evans, D.A., Beckett, L.A., Albert, M.S., Hebert, L.E., Scherr, P.A., Funkenstein, H.H., \& Taylor, J.O. (1993). Level of education and change in cognitive function in a community population of older persons. Annals of Epidemiology, 3, 71-77.

Fabrigoule, C., Letenneur, L., Dartigues, J.F., Zarrouk, M., Commenges, D., \& Barberger-Gateau, P. (1995). Social and leisure activities and risk of dementia: A prospective longitudinal study. Journal of the American Geriatrics Society, 43, 485-490.

Friedland, R.P., Fritsch, T., Smyth, K.A., Koss, E., Lerner, A.J., Chen, C.H., Petot, G.J., \& Debanne, S.M. (2001). Patients with Alzheimer's disease have reduced activities in midlife compared with healthy control-group members. Proceedings of the National Academy of Sciences of the United States of America, 98, 3440-3445.

Friston, K.J., Holmes, A., Price, C., Buchel, C., \& Worsley, K.J. (1999). Multisubject fMRI studies and conjunction analyses. Neuroimage, 10, 385-396.

Grober, E., \& Sliwinski, M. (1991). Development and validation of a model for estimating premorbid verbal intelligence in the elderly. Journal of Clinical and Experimental Neuropsychology, 13, 933-949.

Holmes, A., \& Friston, K. (1998). Generalisability, random effects and population inference. Neuroimage, 7, S754.

Kondo, K., Niino, M., \& Shido, K. (1994). A casecontrol study of Alzheimer's disease in Japan significance of life-styles. Dementia, 5, 314-326.

Letenneur, L., Commenges, D., Dartigues, J.F., \& Barberger-Gateau, P. (1994). Incidence of dementia and Alzheimer's disease in elderly community residents of south-western France. International Journal of Epidemiology, 23, 1256-1261.

Nelson, H.E. (1982). The National Adult Reading Test (NART): Windsor, England, NFER, Nelson.

Nelson, H.E., \& O'Connell, A. (1978). Dementia: The estimation of premorbid intelligence levels using the National Adult Reading Test. Cortex, 14, 234-244.

Price, C.J., \& Friston, K.J. (2001). Functional neuroimaging of cognitive aging. In R. Cabeza \& A. Kingstone (Eds.), Handbook of functional neuroimaging of cognition (pp. 379-399). Cambridge, MA: MIT Press.

Reuter-Lorenz, P.A., Jonides, J., Smith, E.E., Hartley, A., Miller, A., Marshuetz, C., \& Koeppe, R.A. (2000). Age differences in the frontal lateralization of verbal and spatial working memory revealed by PET. Journal of Cognitive Neurosciences, 12, 174-187.

Reuter-Lorenz, P.A., Marshuetz, C., Jonides, J., Smith, E.E., Hartley, A., \& Koeppe, R.A. (2001). Neurocognitive ageing of storage and executive processes. European Journal of Cognitive Psychology, 13, 257-278.

Scarmeas, N., Levy, G., Tang, M.X., Manly, J., \& Stern, Y. (2001). Influence of leisure activity on the incidence of Alzheimer's disease. Neurology, 57, 2236-2242.

Scarmeas, N., Zarahn, E., Anderson, K.E., Habeck, C.G., Hilton, H.J., Flynn, J., Marder, K.S., Bell, K., Sackeim, H.A., Van Heertum, R.L., Moeller, J.R., \& Stern, Y. (2003). Association of leisure activities with cerebral blood flow in Alzheimer's disease: Implications for the cognitive reserve hypothesis. Archives of Neurology, 60, 359-365.

Stern, Y. (2002). What is cognitive reserve? Theory and research application of the reserve concept. Journal of the International Neuropsychological Society, 8 , 448-460.

Stern, Y., Alexander, G.E., Prohovnik, I., \& Mayeux, R. (1992). Inverse relationship between education and parietotemporal perfusion deficit in Alzheimer's disease. Annals of Neurology, 32, 371-375.

Stern, Y., Alexander, G.E., Prohovnik, I., Stricks, L., Link, B., Lennon, M.C., \& Mayeux, R. (1995). Relationship between lifetime occupation and parietal flow: Implications for a reserve against Alzheimer's disease pathology. Neurology, 45, 55-60.

Stern, Y., Gurland, B., Tatemichi, T.K., Tang, M.X., Wilder, D., \& Mayeux, R. (1994). Influence of education and occupation on the incidence of Alzheimer's disease. Journal of the American Medical Association, 271, 1004-1010.

Stern, Y., Moeller, J.R., Anderson, K.E., Luber, B., Zubin, N.R., DiMauro, A.A., Park, A., Campbell, C.E., Marder, K., Bell, K., Van Heertum, R., \& 
Sackeim, H.A. (2000). Different brain networks mediate task performance in normal aging and AD: Defining compensation. Neurology, 55, 1291-1297.

Wechsler, D. (1981). Wechsler Adult Intelligence Scale-Revised. New York, NY: The Psychological Corporation.

White, L., Katzman, R., Losonczy, K., Salive, M., Wallace, R., Berkman, L., Taylor, J., Fillenbaum, G., \& Havlik, R. (1994). Association of education with incidence of cognitive impairment in three established populations for epidemiological studies of the elderly. Journal of Clinical Epidemiology, 47, 363-374.
Wilson, R.S., Mendes De Leon, C.F., Barnes, L.L., Schneider, J.A., Bienias, J.L., Evans, D.A., \& Bennett, D.A. (2002). Participation in cognitively stimulating activities and risk of incident Alzheimer disease. Journal of the American Medical Association, 287, 742-748.

Worsley, K.J., \& Friston, K.J. (1995). Analysis of fMRI time-series revisited-again. Neuroimage, 2, 173-182.

Worsley, K.J., Poline, J.B., Friston, K.J., \& Evans, A.C. (1997). Characterizing the response of PET and fMRI data using multivariate linear models. Neuroimage, 6, 305-319. 